\title{
NUEVA ESPECIE DE Interulobites, ESPORA TRILETE DEL JURÁSICO MEDIO DE LA CUENCA NEUQUINA, ARGENTINA
}

\author{
Marcelo Adrián MARTÍNEZ \\ CONICET (Consejo Nacional de Investigaciones Científicas y Técnicas). \\ Universidad Nacional del Sur. Departamento de Geología. San Juan 670. 8000 \\ -Bahía Blanca, Argentina. E-mail: martinez@criba.edu.ar
}

\begin{abstract}
Martínez, M.A. 2000. Nueva especie de Interulobites, espora trilete del Jurásico Medio de la Cuenca Neuquina, Argentina. [A new species of Interulobites, a trilete spore from the Middle Jurassic of the Neuquén Basin, Argentina.] Revista Española de Paleontología, 15(2), 165-170. ISSN 0213-6937.
\end{abstract}

\begin{abstract}
Interulobites lajensis, a new species of the trilete spore genus Interulobites Phillips, in Phillips and Félix, 1971, is described and figured from the Middle Jurassic (Lajas Formation) of the Neuquén Basin, Argentina. This species is characterized by a trilete-mark generally bordered by lips, which bifurcates near the internal margin of the equatorial thickening (cingulum). Proximal surface granulate and verrucate, and distal surface ornamented by braided rugulae. The accompanying microflora correlates with the Callialasporites dampieri Zone (Late Toarcian - Oxfordian), as defined by Quatrocchio et al. (1996). The presence of ammonoids (Eurycephalites cf. vergarensis) from the Vergarensis Zone (Riccardi et al., 1989), indicates an age-range from Late Bathonian to Early Callovian.
\end{abstract}

Keywords: Trilete spore, Portada Covunco, Lajas Formation, Middle Jurassic, Neuquén Basin, Argentina.

\section{RESUMEN}

Se describe e ilustra Interulobites lajensis, una nueva especie de espora trilete del género Interulobites Phillips, en Phillips y Félix, 1971, correspondiente al Jurásico Medio (Formación Lajas) de la Cuenca Neuquina, Argentina. Interulobites lajensis se caracteriza por presentar los rayos de la marca trilete bifurcados en sus terminaciones, cerca del margen ecuatorial engrosado (cíngulo), generalmente bordeados por labios. Superficie proximal ornamentada con gránulos y verrugas. Superficie distal ornamentada por rúgulas anastomosadas. La microflora analizada se correlaciona con la Zona de Callialasporites dampieri (Toarciense tardío - Oxfordiense), definida por Quattrocchio et al., 1996. La presencia de ammonites (Eurycephalites cf. vergarensis) de la Zona de Vergarensis (Riccardi et al., 1989), indica un rango de edad comprendido entre el Bathoniense tardío y el Calloviense temprano.

Palabras clave: Espora trilete, Portada Covunco, Formación Lajas, Jurásico Medio, Cuenca Neuquina, Argentina.

\section{INTRODUCCIÓN}

Volkheimer y Quattrocchio (1975) mencionan la presencia de Interulobites sp. A, en depósitos correspondientes a la Fm. Vaca Muerta (Tithónico), en el ámbito de la Cuenca Neuquina. Durante la realización de nuevos estudios referentes a la palinoestratigrafía y palinofacies del Jurásico Medio en el sector centrooccidental de la Cuenca Neuquina (Martínez, 1999), se registró la presencia de numerosos ejemplares en muy buen estado de conservación del morfotipo mencionado, lo cual permitió la denominación, caracterización y descripción de una nueva especie: Interulobites lajensis.
El área de estudio (Fig. 1) se localiza $20 \mathrm{~km}$ al noroeste de la ciudad de Zapala, por la ruta nacional $\mathrm{N}^{\circ}$ 22 , a $38^{\circ} 48^{\prime}$ de latitud sur y $70^{\circ} 12^{\prime}$ de longitud oeste. El afloramiento, denominado perfil Portada Covunco, se ubica en el flanco occidental de un anticlinal erosionado, cuyo eje buza hacia el sureste, produciendo un cierre de tipo braquianticlinal. El levantamiento del perfil se inició en los primeros afloramientos del núcleo del anticlinal a 700 metros aguas arriba del puente sobre el Arroyo Covunco y corresponde a Carlos Zavala (en prep.). De los 16 niveles estudiados, se registró la presencia de Interulobites lajensis sp. nov. solamente en el nivel 1602, correspondiente a la Fm. Lajas en facies de sistemas 


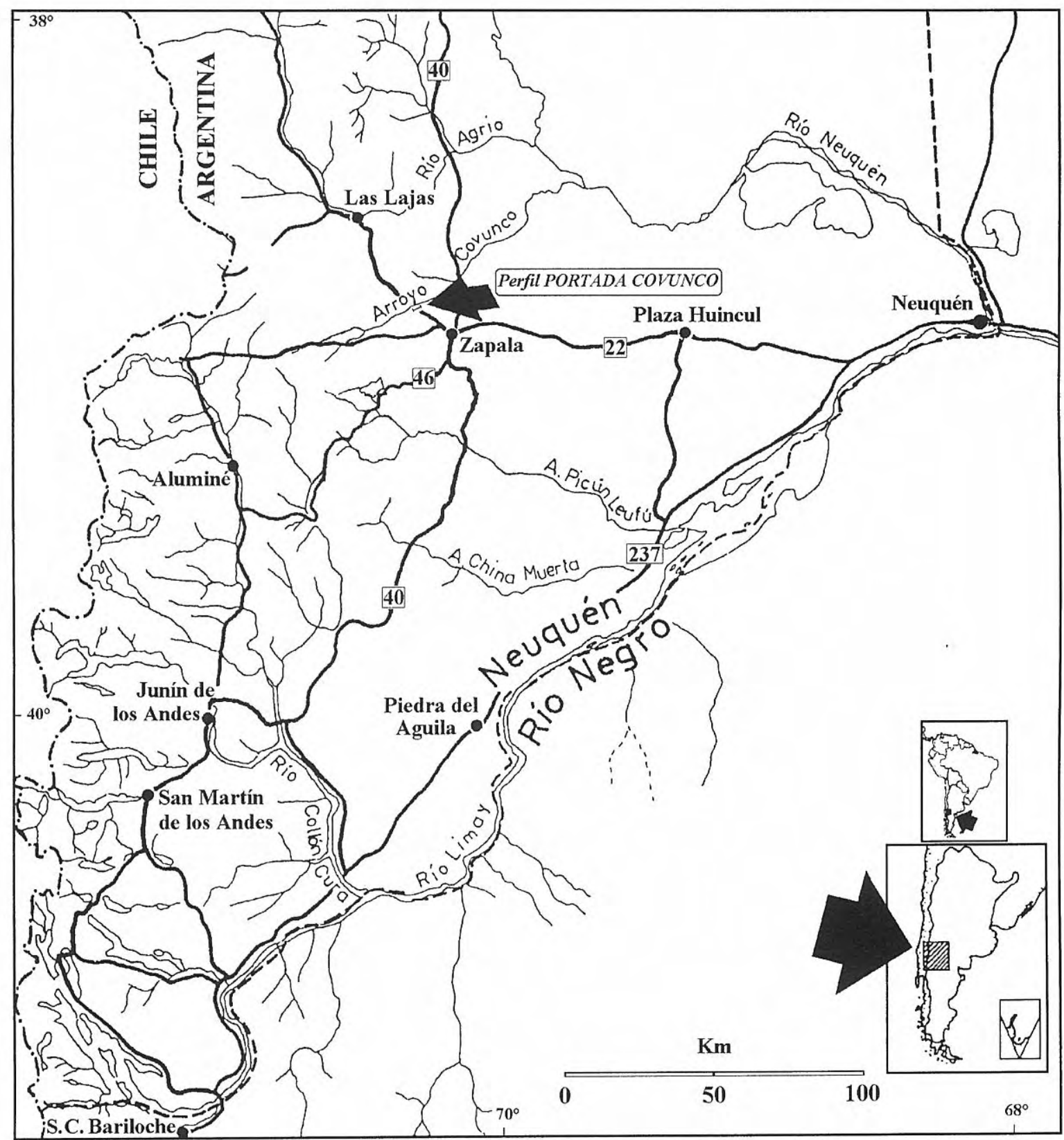

Figura 1. Mapa de ubicación de la provincia de Neuquén y la localidad de estudio. Map of the Province of Neuquén showing the locality studied.

deltaicos modificados por olas. La microflora analizada se correlaciona con la Subzona de Callialasporites turbatus (Toarciense tardío - Bajociense temprano) y la Subzona de Ischyosporites marburgensis (Bajociense temprano alto - Bajociense tardío), ambas pertenecientes a la Zona de Callialasporites dampieri (Toarciense tardío -
Oxfordiense), definidas por Quattrocchio et al. (1996). A los 340 metros de la base, Carlos Gulisano, (com. pers.) reporta el único hallazgo de ammonites: Eurycephalites cf. vergarensis de la Zona de Vergarensis (Riccardi et al., 1989), con un rango de edad comprendido entre el Bathoniense tardío y el Calloviense temprano.

Figura 2. Interulobites lajensis sp. nov. Todas x 1000. A - B. Holotipo. 1602 d: 24/158,9. A, vista proximal; B, vista distal. C. 1602 d: 4,1/138,8. Vista distal. D - E. 1602 d: 2,7/160,6. D, vista proximal; E, vista distal. F. 1602 d: 1,4/145,4. Vista proximal. G. 1602 d: 23/142,1. Vista proximal. H. 1602 c: 10,9/122. Vista proximal. I - J. 1602 e: $18 / 117,7$. I, vista proximal; J, vista distal. K. 1602 d: 21,1/128,4. Vista proximal. L. 1602 d: 24/161,9. Vista proximal. M. 1602 d: 22,3/ 123,5. Vista proximal. N - O. 1602 d: 12,7/104,8. N, vista proximal; O, vista distal. P. 1602 d: 23,6/156. Vista distal.

All x 1000. A-B. Holotype. A, proximal view; B, distal view. 

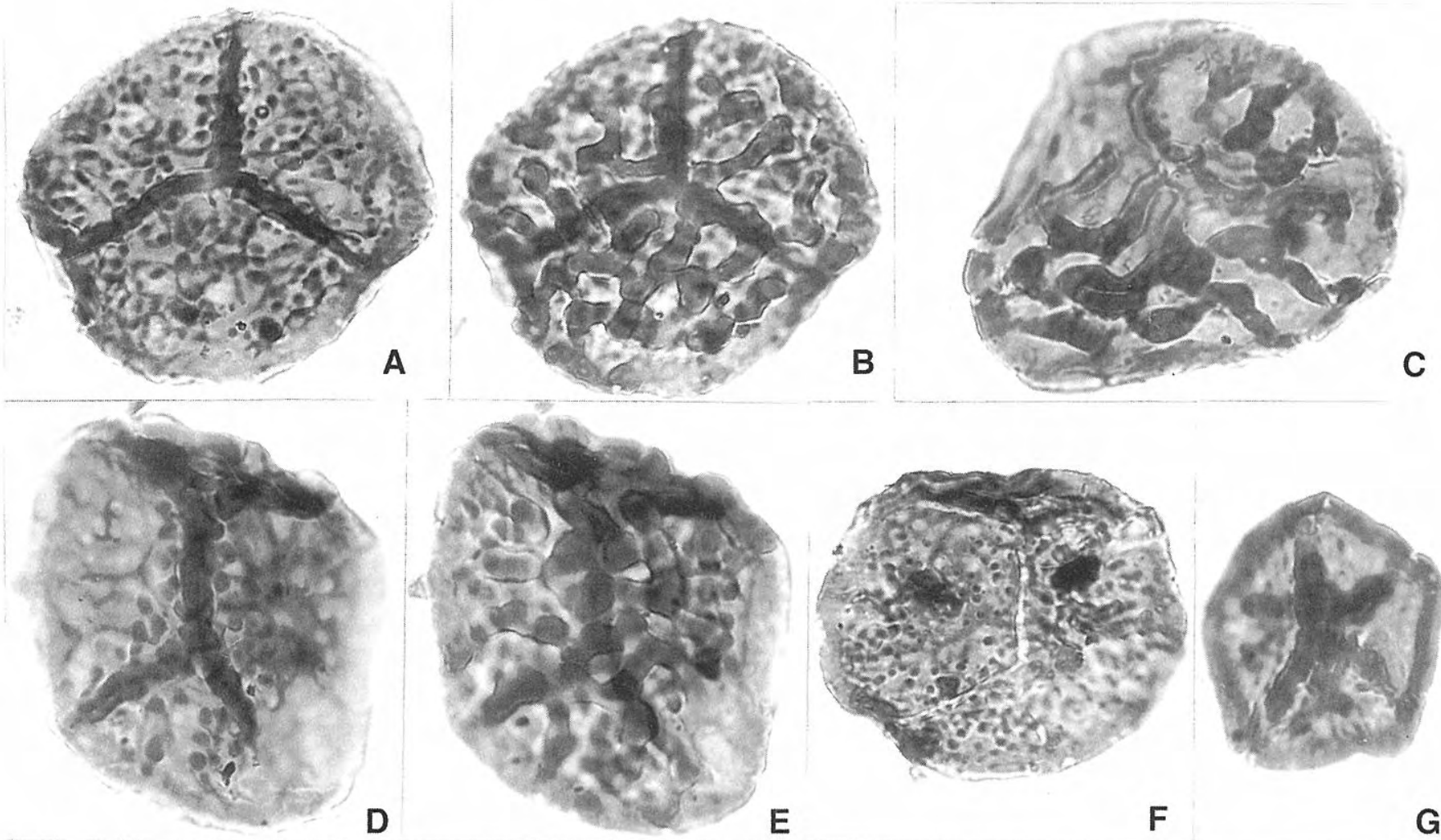

E

$\mathbf{F}$

G
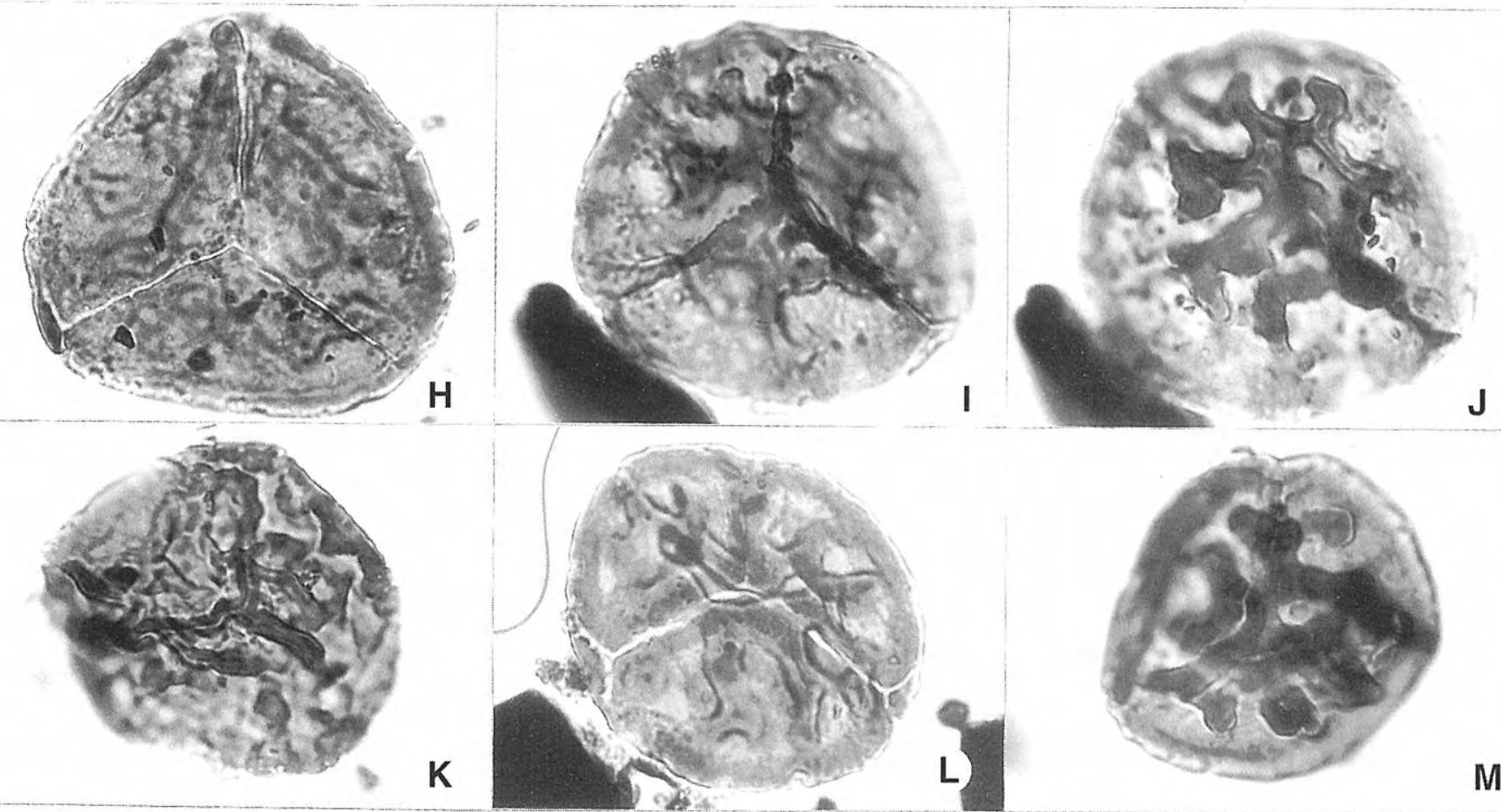

M
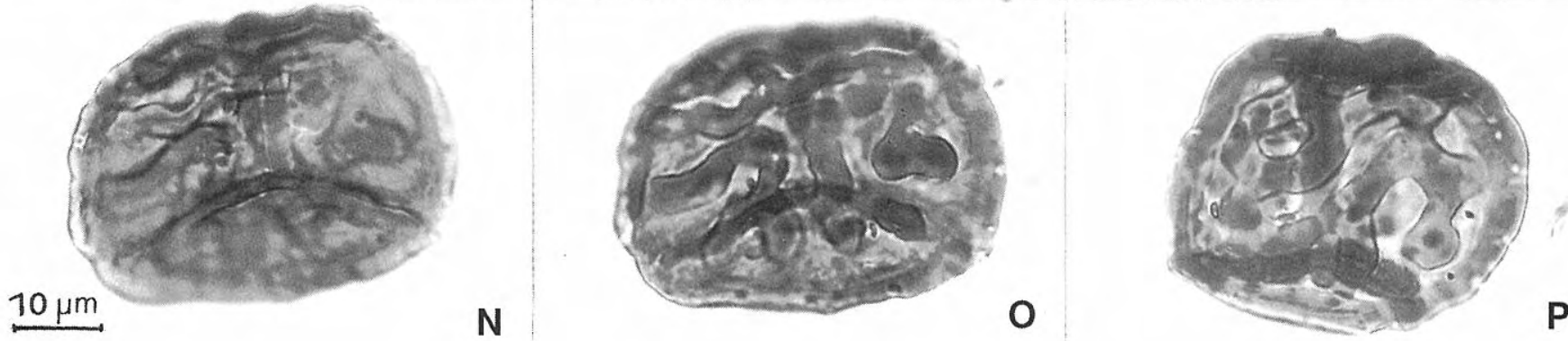


\section{METODOLOGÍA}

El perfil Portada Covunco, de 785 metros de espesor estratigráfico, fue estudiado banco a banco y medido mediante báculo de Jacob (Zavala, en prep.). El material fue obtenido del nivel 1602 (intercalaciones pelíticas de color gris oscuro con delgados bancos de arenisca fina), ubicado a 305,8 metros de la base del perfil.

La extracción físico-química de la muestra palinológica se efectuó sometiendo el material al ataque con ácido clorhídrico $(10 \%)$ en frío para disolver carbonatos, y ácido fluorhídrico concentrado (70\%) en frío, durante 48 horas, para disolver los silicatos. Para el estudio de la asociación microflorística, se trató el residuo con líquidos pesados (cloruro de cinc). Los preparados definitivos se montaron en glicerina gelatina y el residuo palinológico fue preservado agregando solución de formol. Las muestras están archivadas en el Laboratorio de Palinología de la Universidad Nacional del Sur bajo las siglas UNSP-PC .

El estudio palinológico se realizó mediante técnicas de microscopía de luz transmitida, utilizando un microscopio Olympus $\mathrm{BH} 2 \mathrm{~N}^{\circ}$ 100786. Las microfotografías fueron obtenidas con una cámara Olympus y se utilizó película Agfa de 100 ASA (Fig. 2).

El análisis estadístico de la asociación microflorística se realizó a partir de recuentos polínicos (200 palinomorfos), obteniéndose valores de frecuencias relativas (\%) para cada grupo taxonómico.

\section{DESCRIPCIÓN SISTEMÁTICA}

Anteturma SPORITES H. Potonié, 1893

Turma ZONALES Bennie y Kidston, emend. Potonié, 1956

Subturma ZONOTRILETES Waltz, 1935

Infraturma CINGULATI Potonié y Klaus, emend. Dettmann, 1963

\section{Género Interulobites Phillips, en Phillips y Félix, 1971}

Especie tipo: Interulobites intraverrucatus (Brenner, 1963) Phillips y Félix, 1971.

\section{Diagnosis genérica}

P. Phillips (en Phillips y Félix, 1971) propuso el género Interulobites con la siguiente diagnosis genérica (traducida): "Radial, trilete, lesuras simples o bordeadas, llegando al margen interno de un borde ecuatorial engrosado (cíngulo); superficie proximal lisa o con escultura reducida; lado distal fuertemente ornamentado con verrugas, rúgulas o báculas; estos elementos esculturales con superficies internas irregulares se proyectan dentro de la cavidad central de la espora".
1975 Interulobites sp. A Volkheimer y Quattrocchio, 214, lám. III, figs. 1-4.

Derivatio nominis: El nombre hace referencia a la Formación Lajas (Cuenca Neuquina) de la cual proviene la muestra palinológica portadora de esta nueva especie.

Material: UNSP PC. Holotipo: 1602 d: 24/158,9 (Fig. 2AB).

Otros ejemplares ilustrados: 1602 c: 10,9/122 (Fig. 2H); 1602 d: 4,1/138,8 (Fig. 2C); 2,7/160,6 (Fig. 2D-E); 1,4/145,4 (Fig. 2F); 23/142,1 (Fig. 2G); 21,1/128,4 (Fig. 2K); 24/161,9 (Fig. 2L); 22,3/123,5 (Fig. 2M); 12,7/104,8 (Fig. 2N-O); 23,6/156 (Fig. 2P); 1602 e: 18/117,7 (Fig. 2I$\mathrm{J})$.

\section{Diagnosis}

Espora trilete, cingulada, de contorno ecuatorial subcircular hasta ovoidal. Lesuras generalmente bordeadas por labios, bifurcadas en sus terminaciones cerca del ecuador. Superficie distal ornamentada por rúgulas anastomosadas. Superficie proximal ornamentada con gránulos y verrugas de distribución y densidad variable.

Trilete spore, cingulate, subcircular to ovoidal equatorial outline. Laesurae generally bordered by lips, with bifurcate distal endings near to the equatorial margin. Distal surface ornamented with anastomosed rugulae. Proximal surface, granulate to verrucate, with variable distribution and density.

\section{Descripción}

Esporas triletes, de contorno ecuatorial predominantemente subcircular a ovoidal, ocasionalmente polihedral y subtriangular (Fig. 2 D, E, G, H). Los rayos de la marca trilete son rectos a levemente sinuosos (Fig. 2 A, H, K, M), están generalmente bordeados por labios (de hasta $2,5 \mu \mathrm{m}$ de ancho cada uno), y se bifurcan al llegar al borde interno del cíngulo, o bien penetran en el mismo, pero sin alcanzar el margen ecuatorial. El cíngulo es liso o escasamente ornamentado, de ancho uniforme que varía entre 3 y $6 \mu \mathrm{m}$. La cara distal se halla ornamentada por rúgulas de hasta 6 $\mu \mathrm{m}$ de ancho, que se anastomosan para formar un retículo más o menos imperfecto. Esta ornamentación cubre todo el hemisferio distal (Fig. 2 B, C, E, O, P) o bien se concentra en torno al área polar (Fig. 2 G, J). En ocasiones, los muros del retículo tienden a fusionarse con el cíngulo (Fig. 2 B, C). En la cara proximal, puede disponerse una variada ornamentación de gránulos y verrugas subcirculares a ovoidales (de hasta $4 \mu \mathrm{m}$ de diámetro máximo). Algunos ejemplares exhiben facetas proximales con ornamentación más o menos densa y uniformemente distribuída (Fig. 2 A, D, F), otros ejemplares, en cambio, muestran caras proximales con escasa ornamentación a prácticamente lisas (Fig. 2 H, I, L, N).

\section{Dimensiones}

Los rangos de medidas se obtuvieron mediante la medición de hasta 20 ejemplares: 
Holotipo: diámetro ecuatorial: 46 x $53 \mu \mathrm{m}$.

Rasgo medido

$\mathrm{N}^{\circ}$ ej.

medidos

Mín. Máx.

Diámetro ecuatorial mayor

Diámetro ecuatorial menor

18

$42 \mu \mathrm{m} \quad 58 \mu \mathrm{m} \quad 50 \mu \mathrm{m}$

$34 \mu \mathrm{m} \quad 48 \mu \mathrm{m} \quad 42 \mu \mathrm{m}$

Ancho máximo del cíngulo

$20 \quad 3 \mu \mathrm{m} \quad 6 \mu \mathrm{m} \quad 4 \mu \mathrm{m}$

Ancho máximo de c/ labio

16

$$
1 \mu \mathrm{m} \quad 2,5 \mu \mathrm{m} \quad 2 \mu \mathrm{m}
$$

\section{Comparaciones}

En la Tabla 1 se presentan las características de las especies afines a Interulobites lajensis sp. nov.: I. intraverrucatus (Brenner, 1963) Phillips y Félix (1971), en el sentido de Scott (1976); I. sinuosus Scott, 1976 e I. algoensis Scott, 1976; todas citadas por Scott (1976) para depósitos del Cretácico temprano, de la Cuenca de Algoa en Sudáfrica. I. pseudoreticulatus Archangelsky, 1983 e Interulobites sp. Archangelsky, 1983, citadas en Archangelsky et al. (1983) para el Cretácico de Argentina austral.

\section{Observaciones}

En el nivel estudiado, I. lajensis se presenta con una frecuencia de $8,70 \%$. La asociación microflorística se caracteriza por el predominio de esporas (40\%), siendo especies frecuentes Nevesisporites cf. radiatus $(7,83 \%)$, Taurocusporites sp. (6,09\%), Antulsporites saevus (1,30\%) y Deltoidospora minor $(1,30 \%)$; Cheirolepidiaceae (40\%), con Classopollis simplex $(18,26 \%)$ y Classopollis cf. classoides $(11,30 \%)$ como taxones más abundantes, y Podocarpaceae (10\%) representadas por Microcachryidites castellanosii (1,30\%), Podocarpidites sp. cf. $P$. verrucosus $(0,87 \%)$ y Callialasporites dampieri $(0,87 \%)$, entre otros.

\section{Distribución estratigráfica}

Interulobites sp. A fue citado para la Fm. Vaca Muerta (Tithónico) en la Cuenca Neuquina (en Volkheimer y Quattrocchio, 1975).

\section{AGRADECIMIENTOS}

El autor desea expresar su agradecimiento a la Dra. Mirta E. Quattrocchio por la lectura crítica del manuscrito, al Dr. Carlos Zavala por sus interpretaciones en el trabajo de campo, al Consejo Nacional de Investigaciones Científicas y Técnicas (CONICET), al Departamento de Geología y a la Secretaría General de Ciencia y Técnica (SGCyT) de la Universidad Nacional del Sur, por el apoyo brindado; así como también a los árbitros por la lectura y sugerencias recibidas oportunamente.

\begin{tabular}{|c|c|c|c|c|c|c|}
\hline $\begin{array}{c}\text { Rasgo } \\
\text { observado }\end{array}$ & $\begin{array}{c}\text { I. intraverrucatus } \\
\text { (Brenner, 1963) Phillips } \\
\text { \& Félix, 1971 }\end{array}$ & $\begin{array}{l}\text { I. sinuosus } \\
\text { Scott, } 1976\end{array}$ & $\begin{array}{l}\text { I. algoensis } \\
\text { Scott, } 1976\end{array}$ & $\begin{array}{l}\text { I. pseudoreticulatus } \\
\text { Archangelsky, } 1983\end{array}$ & $\begin{array}{c}\text { I. sp. } \\
\text { Archangelsky, } 1983\end{array}$ & $\begin{array}{l}\text { I. lajensis sp. nov } \\
\text { (en este trabajo) }\end{array}$ \\
\hline $\begin{array}{l}\text { Contorno } \\
\text { Ecuatorial }\end{array}$ & circular a subtriangular & circular a subtriangular & \pm circular & $\begin{array}{l}\text { triangular ángulos } \\
\text { redondeados }\end{array}$ & subcircular & $\begin{array}{c}\text { subcircular a ovoidal } \\
\text { ocasionalmente } \\
\text { polihedral y } \\
\text { subtriangular }\end{array}$ \\
\hline $\begin{array}{l}\text { Diámetro } \\
\text { Ecuatorial }\end{array}$ & $30-(34)-43 \mu \mathrm{m}$ & $37-47 \mu \mathrm{m}$ & $38-(46)-53 \mu \mathrm{m}$ & $42-(57)-64 \mu \mathrm{m}$ & $30-50 \mu \mathrm{m}$ & $42-(50)-58 \mu \mathrm{m}$ \\
\hline Lesuras & alcanzan el ecuador & $\begin{array}{l}\text { alcanzan el margen } \\
\text { interno del cíngulo }\end{array}$ & $\begin{array}{c}\text { rectas, elevadas, } \\
\text { levemente onduladas } \\
\text { alcanzando el ecuador }\end{array}$ & $\begin{array}{l}\text { rectas a muy levemente } \\
\text { sinuosas, alcanzan el } \\
\text { margen del cíngulo }\end{array}$ & $\begin{array}{l}\text { alcanzan el borde } \\
\text { interno del cíngulo }\end{array}$ & $\begin{array}{l}\text { se bifurcan (en el borde } \\
\text { interno del cíngulo o } \\
\text { dentro del mismo) sin } \\
\text { alcanzar el margen } \\
\text { ecuatorial }\end{array}$ \\
\hline $\begin{array}{l}\text { Ancho } \\
\text { de los } \\
\text { labios }\end{array}$ & $1-2,5 \mu \mathrm{m}$ & $2-3 \mu \mathrm{m}$ & $\begin{array}{c}1,5 \mu \mathrm{m} \text { (por mayor } \\
\text { concentración de } \\
\text { ornamentación) }\end{array}$ & $\begin{array}{l}\text { entero }(3-5 \mu \mathrm{m}) \text {, o por } \\
\text { mayor concentración de } \\
\text { ornamentación) }\end{array}$ & entero & $\begin{array}{c}\text { generalmente presentes, } \\
1-(2)-2,5 \mu \mathrm{m} \text { cada } \\
\text { uno. }\end{array}$ \\
\hline $\begin{array}{l}\text { Cara } \\
\text { distal }\end{array}$ & $\begin{array}{l}\text { reticulado a foveo- } \\
\text { reticulado, con muros de } \\
2-4 \mu \mathrm{m} \text { de ancho y } \\
\text { lúminas de } 2-5 \mu \mathrm{m} \text {. } \\
\text { Los muros se unen al } \\
\text { cíngulo } \\
\end{array}$ & $\begin{array}{c}\text { crestas amplias, } \\
\text { prominentes y curvadas } \\
\text { de } 4-7 \mu \mathrm{m} \text { de ancho, } \\
\text { que no se unen al } \\
\text { cíngulo }\end{array}$ & $\begin{array}{l}\text { verrugas y rúgulas bajas } \\
\text { que a veces se unen y } \\
\text { forman un reticulo. Los } \\
\text { muros }(3-4 \mu \mathrm{m} \text { de } \\
\text { ancho) llevan espinas } \\
(0,8 \times 1 \mu \mathrm{m})\end{array}$ & $\begin{array}{l}\text { muros sinuosos, } \pm \\
\text { fusionados, formando o } \\
\text { no un reticulo irregular; } \\
\text { hacia el ecuador } \\
\text { aparecen verrugas entre } \\
\text { muros gruesos }\end{array}$ & $\begin{array}{l}\text { grandes verrugas de } \\
\text { diámetro variable, } \\
\text { juntas, circulares o } \\
\text { alargadas }\end{array}$ & $\begin{array}{c}\text { rúgulas de hasta } 6 \mu \mathrm{m} \text { de } \\
\text { ancho que se } \\
\text { anastomosan para } \\
\text { formar un reticulo } \pm \\
\text { imperfecto }\end{array}$ \\
\hline $\begin{array}{c}\text { Cara } \\
\text { proximal }\end{array}$ & $\begin{array}{l}\text { rugulado a verrucado, } \\
\text { con elementos de } \\
1,5-2,5 \mu \mathrm{m} \text { de ancho } \\
\text { y muros de } 3 \mu \mathrm{m} \text { de } \\
\text { ancho paralelos } \\
\text { a las lesuras }\end{array}$ & $\begin{array}{l}\text { algunas verrugas de } \\
1-3 \mu \mathrm{m} \text { de ancho }\end{array}$ & $\begin{array}{c}\text { Algunos gránulos y } \\
\text { verrugas de hasta } 2 \mu \mathrm{m} \\
\text { de ancho, no muy } \\
\text { densamente distribuídos }\end{array}$ & $\begin{array}{c}\text { lisa o con escasos granos } \\
\text { y verrugas }\end{array}$ & $\begin{array}{l}\text { pequeñas verrugas, } \\
\text { uniformemente } \\
\text { distribuídas, separadas } \\
\text { entre sí por una } \\
\text { distancia } \geq \text { que su } \\
\text { diámetro basal } \\
\end{array}$ & $\begin{array}{l}\text { gránulos y verrugas } \\
\text { subcirculares a ovoidales } \\
\text { (hasta } 4 \mu \mathrm{m} \text { de diám. } \\
\text { máximo) de variada } \\
\text { distribución y densidad }\end{array}$ \\
\hline $\begin{array}{l}\text { Ancho } \\
\text { del } \\
\text { cíngulo }\end{array}$ & $\begin{array}{c}3-5 \mu \mathrm{m} \text {, borde externo } \\
\text { difuso }\end{array}$ & $5-8 \mu \mathrm{m}$ & $3-6 \mu \mathrm{m}$, con espinas & $2,5-7 \mu \mathrm{m}$ & $2,5-5 \mu \mathrm{m}$ & $\begin{array}{l}\text { liso o escasamente } \\
\text { ornamentado, } \\
3-(4)-6 \mu \mathrm{m} \text {. }\end{array}$ \\
\hline
\end{tabular}

Tabla 1. Cuadro comparativo entre Interulobites intraverrucatus, I. sinuosus, I. algoensis, I. pseudoreticulatus, I. sp. e I. lajensis sp. nov.

Comparison between Interulobites intraverrucatus, I. sinuosus, I. algoensis, I. pseudoreticulatus, I. $s p$. and I. lajensis sp. nov. 


\section{BIBLIOGRAFÍA}

Archangelsky, S., Baldoni, A., Gamerro, J.C. y Seiler, J. 1983. Palinología estratigráfica del Cretácico de Argentina austral. II. Descripciones sistemáticas. Ameghiniana, 20, 199-226.

Martínez, M.A., 1999. Palinología Estratigráfica del Jurásico del centro-oeste de la Cuenca Neuquina. Tesis Doctoral inédita. Universidad Nacional del Sur. 238 pp., Bahía Blanca, Argentina.

Phillips, P. and Félix, J. 1971. A study of Lower and Middle Cretaceous spores and pollen from the Southeastern United States. I. Spores. Pollen et Spores, 13, 279-348.

Quattrocchio, M.E., Sarjeant, W.A.S. and Volkheimer, W. 1996. Marine and terrestrial Jurassic microfloras of
Neuquén Basin (Argentina): Palynological Zonation. In: Advances in Jurassic research (Ed. A.C. Riccardi). Transtec Publications, Switzerland. GeoResearch Forum, Vols. 1-2, 167-178.

Riccardi, A.C., Westermann, G.E.G. and Elmi, S. 1989. The Middle Jurassic Bathonian-Callovian Ammonite Zones of the Argentine-Chilean Andes. Geobios 22, 553-597.

Scott, L. 1976. Palynology of Lower Cretaceous deposits from the Algoa Basin (Republic of South Africa). Pollen et Spores, 18, 563-609.

Volkheimer, W. y Quattrocchio, M. 1975. Palinología estratigráfica del Titoniano (Formación Vaca Muerta) en el área de Caichigüe (Cuenca Neuquina). Parte A. Especies terrestres. Ameghiniana, 12, 193-241.

Manuscrito recibido: 10 de septiembre, 1999 Manuscrito aceptado: 10 de diciembre, 1999 